\title{
EXPANDED CLINICAL SPECTRUM OF MULTIPLE EVANESCENT WHITE DOT SYNDROME WITH MULTIMODAL IMAGING
}

\author{
MARCELA MARSIGLIA, MD, PhD, $* \dagger$ ROBERTO GALLEGO-PINAZO, MD, PHD, \\ EDUARDO CUNHA DE SOUZA, MD, $\S$ MARION R. MUNK, MD, đ** SUQUIN YU, MD, $\dagger \dagger$ \\ SARAH MREJEN, MD, + + EMMETT T. CUNNINGHAM, JR., MD, PHD, §§ BRANDON J. LUJAN, MD, III \\ NAOMI R. GOLDBERG, MD, PHD, $* * *$ THOMAS A. ALBINI, MD, $\dagger \dagger$ ALAIN GAUDRIC, MD, $+\$$

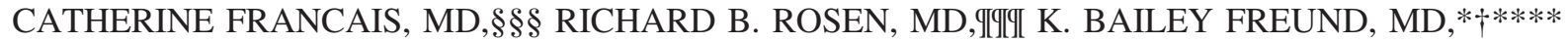 \\ LEE M. JAMPOL, MD,I LAWRENCE A. YANNUZZI, MD*†****
}

Purpose: To evaluate and characterize multiple evanescent white dot syndrome abnormalities with modern multimodal imaging modalities.

Methods: This retrospective cohort study evaluated fundus photography, fluorescein angiography, indocyanine green angiography, optical coherence tomography, enhanced depth imaging optical coherence tomography, short-wavelength autofluorescence, and near-infrared autofluorescence.

Results: Thirty-four multiple evanescent white dot syndrome patients with mean age of 28.7 years were studied (range, 14-49 years). Twenty-six patients were women, and eight were men. Initial mean visual acuity was $0.41 \log M A R$. Final mean visual acuity was 0.03 logMAR. Fluorescein angiography shows a variable number of mid retinal early fluorescent dots distributed in a wreathlike pattern, which correlate to fundus photography, fundus autofluorescence, and indocyanine green angiography. Indocyanine green angiography imaging shows the dots and also hypofluorescent, deeper, and larger spots, which are occasionally confluent, demonstrating a large plaque of deep retinal hypofluorescence. Optical coherence tomography imaging shows multifocal debris centered at and around the ellipsoid layer, corresponding to the location of spots seen with photography, indocyanine green angiography, and fluorescein angiography. Protrusions of the hyperreflectant material from the ellipsoid layer toward the outer nuclear layer correspond to the location of dots seen with photography, indocyanine green angiography, and fluorescein angiography.

Conclusion: Multimodal imaging analysis of the retina in patients with multiple evanescent white dot syndrome shows additional features that may help in the diagnosis of the disease and in further understanding its etiology. Multiple evanescent white dot syndrome is predominantly a disease of the outer retina, centered at the ellipsoid zone, but also involving the interdigitation zone and the outer nuclear layer.

RETINA 36:64-74, 2016

$\mathrm{M}$ ultiple evanescent white dot syndrome (MEWDS) was first described in 1984 by Jampol et al. ${ }^{1}$ Young healthy adults, predominantly females, were noted to experience unilateral diminished vision and enlargement of the blind spot. The retinal findings in these cases included multifocal, small white lesions concentrated not only in the paramacular area but also in the midperipheral fundus (Figure 1). These lesions were presumed to be located in the deep retina or retinal pigment epithelium (RPE). Macular granularity, disk edema (Figure 1), and posterior vitreous cells were seen in many cases. ${ }^{1}$ The mean duration of the disease was 7.5 weeks with spontaneous resolution of the retinal manifestations and improvement of the visual acuity to a normal level. ${ }^{1}$

At the time of its description, only fluorescein angiography (FA) was available as a diagnostic imaging adjunct. During the acute stage of the disease, 


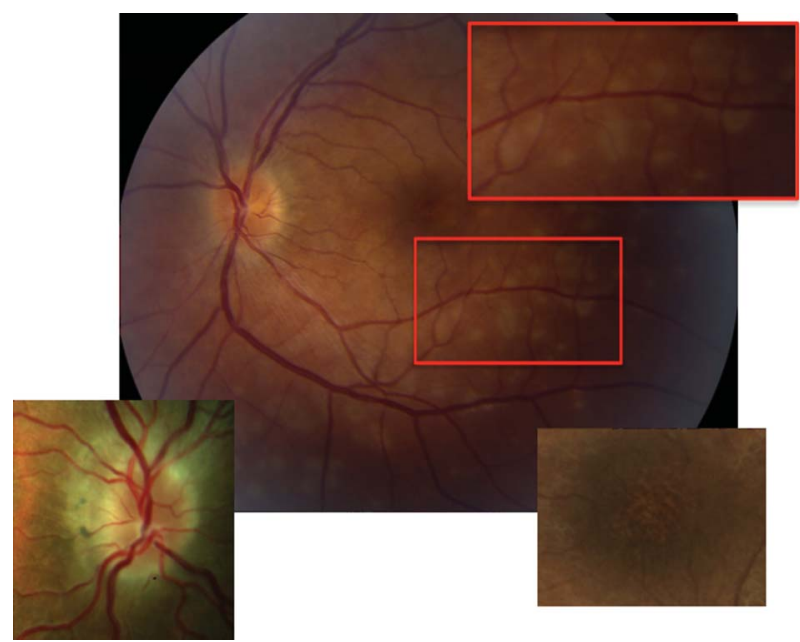

Fig. 1. Color photography findings. Macular granularity, disk edema, and multifocal white lesions concentrated not only in the paramacular area but also in the midperipheral fundus.

early hyperfluorescence with minimal late staining was evident at the site of the lesions (Figure 2). When the lesions resolved, FA showed little evidence of any permanent abnormality and the vision improved. ${ }^{1}$

Subsequent development of new imaging technology has added further observations. In 1996, Obana et $\mathrm{al}^{2}$ reported the indocyanine green angiography (ICGA) findings in 4 cases of MEWDS showing no abnormal signs in the early phase of the angiogram, with hypofluorescence of the lesions in the late phases in the posterior pole and the midperipheral fundus (Figure 3). A greater number of lesions were noted with ICGA

From the *Vitreous-Retina-Macula Consultants of New York and the LuEsther T. Mertz Retinal Research Center, Manhattan Eye, Ear, and Throat Institute, New York, New York; †Department of Ophthalmology, Columbia University, New York, New York; $\ddagger$ Department of Ophthalmology, University and Polytechnic Hospital La Fe, Valencia, Spain; §Faculdade de Medicina da Universidade de São Paulo (FMUSP), Sao Paulo, Brazil; I[Department of Ophthalmology, Northwestern University, Feinberg School of Medicine, Chicago, Illinois; **Department of Ophthalmology, Inselspital, University Hospital Bern, Bern, Switzerland; $†$ Department of Ophthalmology, Shanghai Jiao Tong University Affiliated First People's Hospital, Shanghai, China; $\$$ Quinze-Vingts Hospital, DHU ViewMaintain, Paris, France; $\S \S$ Department of Ophthalmology, California Pacific Medical Center, San Francisco, California; IIITWest Coast Retina Medical Group, San Francisco, California; ***Department of Ophthalmology, The Icahn School of Medicine at Mount Sinai, New York, New York; ††Department of Ophthalmology, Bascom Palmer Eye Institute, Miami, Florida; $\$+\$$ Hospital Lariboisiere, Uni-

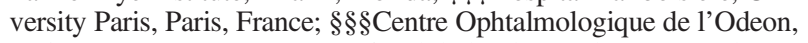

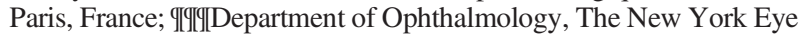
and Ear Infirmary, New York, New York; and *****Department of Ophthalmology, New York University, New York, New York.

Supported by The Macula Foundation, Inc.

None of the authors have any financial/conflicting interests to disclose.

Reprint requests: Marcela Marsiglia, MD, PhD, 210 East 64th Street, 8th Floor, LuEsther T. Mertz Retinal Research Center, Manhattan Eye, Ear and Throat Hospital, New York, NY 10021; e-mail: marcelamarsiglia@hotmail.com than were evident clinically. The ICGA changes disappeared at the recovery stage of the disease.

Gross et $\mathrm{al}^{3}$ classified the lesions seen on FA and ICGA as dots and spots, two components seen in some but not all cases. The dots corresponded to small lesions seen in the fundus ( $\sim 100$ microns) and exhibited an incomplete "wreath" configuration in the early phase of the FA. The late phase of the FA of some patients showed the dots as a diffuse hyperfluorescence in the macula (Figure 2). The lesions seen on ICGA corresponded to the wreathlike patterns. In some areas, the ICGA showed larger, more posterior hypofluorescent abnormalities (>200 microns) referred to as spots (Figure 3 ). The spots also stained slightly in the late phases of the FA but remained hypofluorescent on the ICGA study. Some confluent spots generated larger zonal areas of hypofluorescence (Figure 3). In 2007, Nguyen et al first used optical coherence tomography (OCT) to describe the microstructural abnormalities in MEWDS. They noted subtle disruptions of the inner/outer segment junction, today known as the ellipsoid zone. ${ }^{4}$

In 2008, Yenerel et al first described short-wavelength fundus autofluorescence (SW-AF) findings in MEWDS. The lesions were hyperautofluorescent, even when not visible clinically. ${ }^{5}$ Later, Furino et $\mathrm{al}^{6}$ showed that the hyperautofluorescent lesions in SW-AF corresponded precisely to the site of the focal hypofluorescence seen on late ICGA images; however, more numerous hypofluorescent lesions were seen with the ICGA than hyperautofluorescent lesions with SW-AF. Further publications reported variations in the clinical presentation and course of MEWDS. To date, at least 22 cases of bilateral involvement have been described with no apparent difference from unilateral cases. ${ }^{1,4,7-22}$ Secondary choroidal neovascularization has been reported, ${ }^{23}$ but only one case was described with choroidal neovascularization secondary to a typical MEWDS clinical presentation. $^{24}$ Recurrent episodes (one or more) have also been reported in 12 cases of MEWDS. 7,8,15,18,25,26

A resemblance to other so-called "white spot syndromes," particularly idiopathic multifocal choroiditis (MFC), has been reported in the ophthalmic literature. $^{23,27}$ However, with recognition that MEWDS itself may cause chorioretinal scars and that MFC may have transient acute white lesions that disappear without scars, the use of multimodal imaging (MMI) is necessary to determine the definitive diagnosis. In this era of MMI, MEWDS has been studied by our collaborative group with current technology. The purpose of this study was to investigate imaging abnormalities to enhance our understanding of the precise nature of the clinical manifestations and to improve our understanding of its pathogenesis. 
Fig. 2. Fluorescein angiography findings. Early hyperfluorescence with minimal late staining is evident at the site of the lesions. Small lesions $(\sim 100$ microns) with an incomplete "wreath" configuration in the early phase of the FA correspond to the dots. Optic nerve leakage and window defect in the foveal area were also observed.

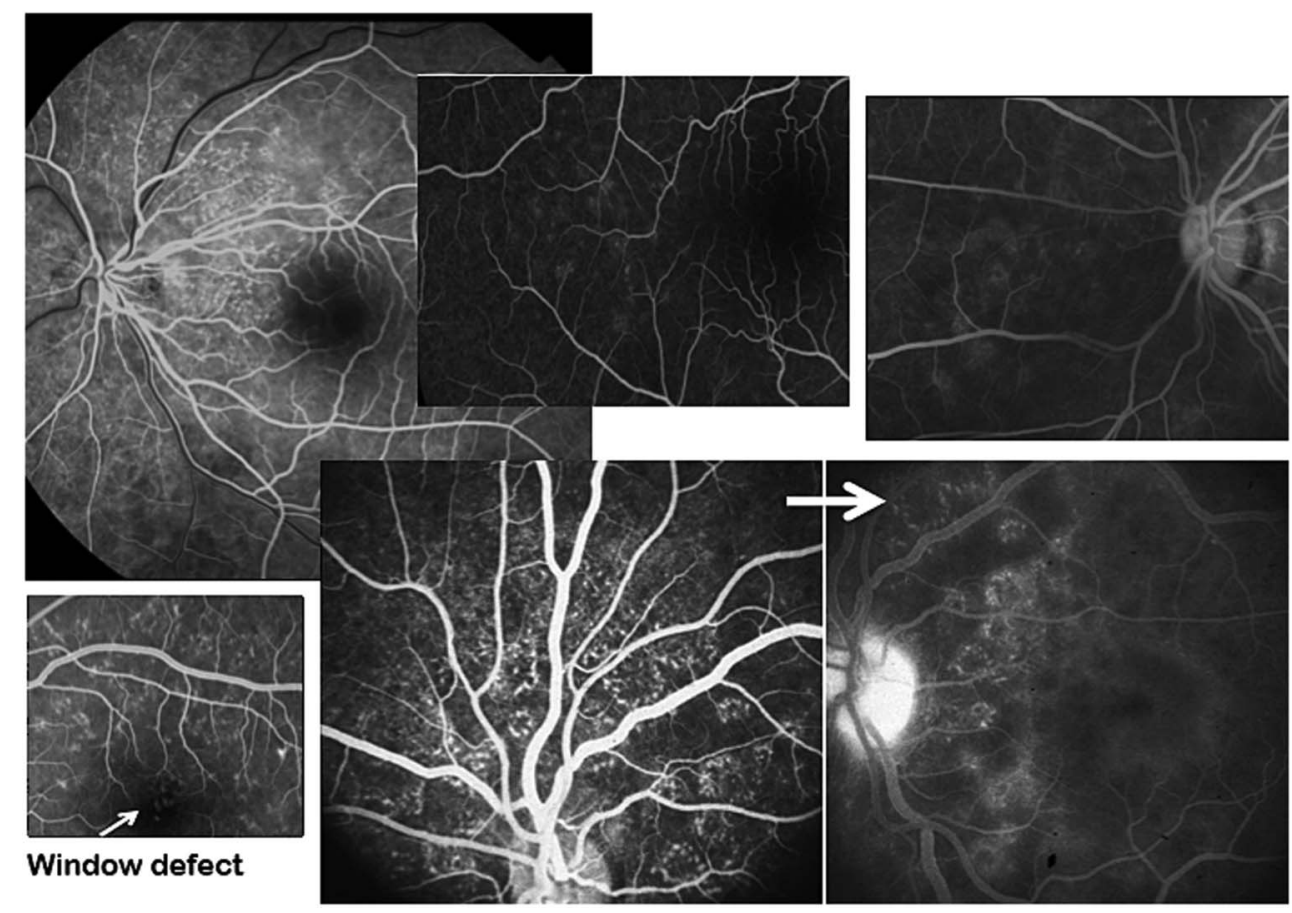

\section{Methods}

This is a retrospective cohort study, The MEWDS cases were collected at two centers by experienced retinal specialists using MMI systems in their patients. This included cases seen at Vitreous-Retina-Macula
Consultants of New York, Northwestern University, or through mail consultation. All imaging results were reviewed by the authors. Disagreements in interpretation were reconciled by one of the authors (L.A.Y.). Only patients who showed the classic findings of this disease as described in the ophthalmic literature were
Fig. 3. Indocyanine green angiography findings. Dots, spots, and dots on spots were observed on ICGA. The spots were larger, more posterior hypofluorescent abnormalities (>200 microns) and remained hypofluorescent in the late phases of the ICGA study. Some confluent spots generated larger zonal areas of hypofluorescence. The dots were more superficial, smaller, and more intensely hypofluorescent.

\section{Dots on Spots}

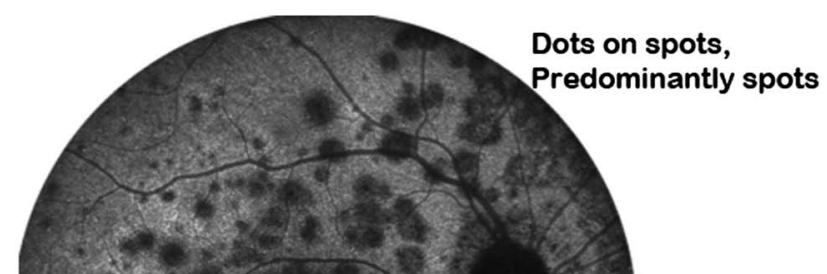

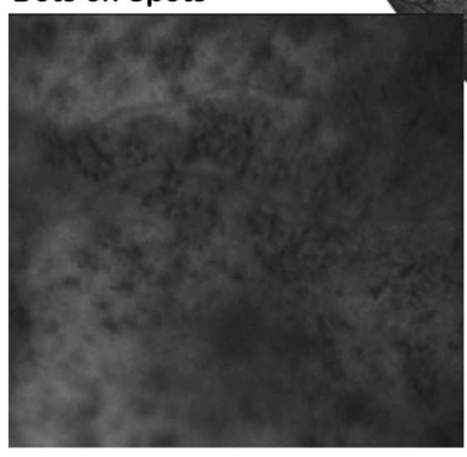

Dots on Spots

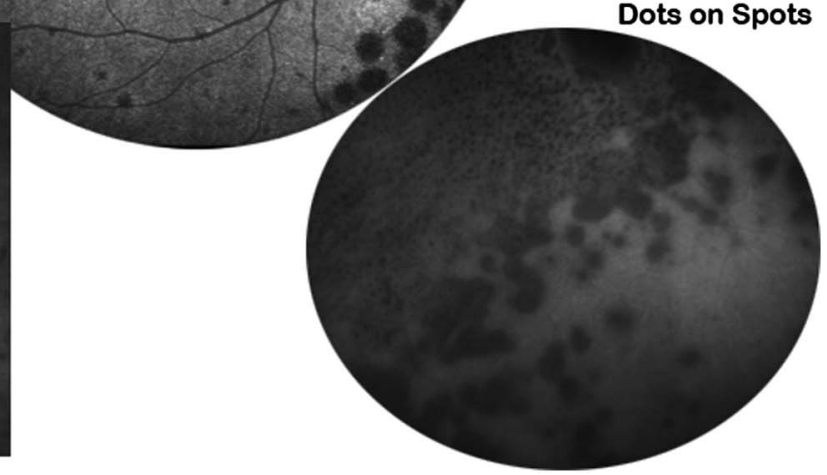


included. ${ }^{1}$ A total of 34 primary cases were identified with characteristics typical of MEWDS and its variant secondary manifestations, such as recurrences, chorioretinal focal scars, and peripapillary degeneration. The study adhered to the Health Insurance Portability and Accountability Act of 1996 and followed the tenets of the Declaration of Helsinki. This study was approved by the North Shore-LIJ Heath System Institutional Review Board, Long Island, NY, and by the Northwestern University Institutional Review Board.

\section{Fundus Photography}

Color and monochromatic red-free photographs were acquired using a Topcon TRC 501X fundus camera (Topcon Medical Systems, Tokyo, Japan). Near-infrared reflectance images were acquired with the Heidelberg Spectralis system (HRA Heidelberg Engineering, Heidelberg Germany) using a $30^{\circ}$ square field of view at a resolution of 1,536 square pixels after standard procedure for the image acquisition including focus of the retinal image in the infrared reflection mode with an excitation wavelength of $820 \mathrm{~nm}$.

\section{Fluorescein Angiography}

Fluorescein angiography was obtained after intravenous injection of $10 \mathrm{~mL}$ of $5 \%$ fluorescein dye using a Topcon TRC 501X fundus camera (Topcon Medical Systems) or a confocal scanning laser ophthalmoscope (Spectralis HRA + OCT; Heidelberg Engineering, Heidelberg, Germany).

\section{Indocyanine Green Angiography}

Indocyanine green angiography images were acquired after intravenous injection of $50 \mathrm{mg}$ of indocyanine green dye using a Topcon TRC 501X fundus camera (Topcon Medical Systems) or a confocal scanning laser ophthalmoscope (Spectralis HRA + OCT; Heidelberg Engineering).

\section{Spectral Domain Optical Coherence Tomography}

Optical coherence tomography imaging was performed with the Spectralis HRA + OCT (Heidelberg Engineering) in 24 studied patients. This equipment provided simultaneous OCT scans and near-infrared reflectance, SW-AF, FA, or ICGA imaging. Subsequent image superimposition allowed point-to-point correlation between the en face and cross-sectional images. The OCT imaging was acquired with a broadband 870-nm superluminescent diode that scanned the retina at 40,000 A-scans per second with an optical axial depth resolution of $7 \mu \mathrm{m}$. The standard protocol included at least 12 OCT scans averaged to reduce signal-to-noise ratio by a factor of 5 and at least one 9-mm horizontal line scan through the fovea. Four patients were imaged with Cirrus HD-OCT in the macular cube mode of $200 \times 200$ scans (Carl Zeiss Inc, Thornwood, NY), one with Optovue Avanti, RTVue XR wide-field en face OCT (Optovue, Fremont, CA), and one with Stratus OCT (Carl Zeiss Meditec, Inc, Dublin, CA). Enhanced depth imaging OCT was acquired following the methodology previously described in the literature. ${ }^{28,29}$

En face OCT analysis was performed in a total of six patients. Two patients were imaged with the Spectralis device, three with the Cirrus OCT, and one with the Spectralis and with the Optovue OCT systems. The thickness of cuts for the en face analysis was $\sim 18$ microns for the choriocapillaris, RPE, ellipsoid zone, and the outer portion of the outer nuclear layer (ONL). A cut of 40 microns was used to study the midchoroid. Optical coherence tomography angiography was performed in one case with the Optovue system. Only five patients did not have OCT imaging, and some of the patients were imaged with more than one system.

\section{Short-Wavelength Fundus Autofluorescence}

Short-wavelength autofluorescence imaging was performed with a confocal scanning laser ophthalmoscope (Spectralis HRA + OCT; Heidelberg Engineering) using a $30^{\circ}$ square field of view at a resolution of 1,536 square pixels. Images were obtained with an optically pumped solid-state laser with an excitation wavelength of $488 \mathrm{~nm}$ and a barrier filter of $495 \mathrm{~nm}$ used to modulate the blue argon excitation light after standard procedure for imaging acquisition including focus of the retinal image. At least nine single images encompassing the entire macular area with at least a portion of the optic disk were acquired and computationally averaged to produce a single frame with improved signal-to -noise ratio.

\section{Near-Infrared Fundus Autofluorescence}

Near-infrared fundus autofluorescence $30^{\circ}$ images were acquired with the HRA2 (Heidelberg Engineering) in the ICGA mode (without dye injection) with an excitation wavelength of $787 \mathrm{~nm}$.

\section{Results}

\section{Patients}

A total of 34 MEWDS patients with mean age of 28.7 years were included in this study (range, 14-49 years). Of these patients, 26 were women and 8 were 
men. Initial mean visual acuity was $0.41 \log M A R$. Final mean visual acuity was $0.03 \log$ MAR. Twenty of the study patients had clinically evident MEWDS in their left eye, and 14 in their right eye. Eighteen subjects were myopic, and three were emmetropic. The refraction information was not available for 13 patients.

Preceding the onset of MEWDS, 3 individuals had a flulike episode, one had received flu vaccination 4 weeks ago, one had a contraceptive ring implanted 4 days ago, one reported nausea and headache, one had gastroenteritis a few days ago, 1 had Hashimoto thyroiditis and a history of anterior uveitis over 30 years ago, one had the diagnosis of $\operatorname{IgG} 2$ and $\mathrm{IgG} 4$ deficiency, and 2 patients suffered from an anxiety disorder. Photopsia was one of the initial complaints in 15 cases. Central vision loss was described in 27 cases, and a visual field defect (usually temporal) was detected in 13 cases. One subject had two previous episodes of MEWDS in the fellow eye, one subject had one recurrence in the same eye, one subject had one previous episode of MEWDS resembling idiopathic MFC in the fellow eye, and one patient had two previous episodes of MEWDS resembling idiopathic MFC in the study eye.

\section{Multimodal Imaging}

The imaging modalities were analyzed for each of the study subjects. Color fundus photography was available in 32 cases, near-infrared reflectance in 29, FA in 29, ICGA in 22, OCT in 29, enhanced depth imaging OCT in 11, SW-AF in 29, NIR-AF in 4 , and en face OCT imaging in 7 cases.

Fundus photography. Color pictures showed multifocal lesions in 29 cases, foveal granularity in 32 cases, and optic nerve edema in 27 cases (Figure 1). Foveal granularity was also evident in other modalities, such as red-free photography, and near-infrared reflectance. The multifocal lesions were best seen with red-free images.

Fluorescein angiography. The FA showed the typical multifocal white spots to be hyperfluorescent in the early stages of the angiogram. These lesions appeared localized to the middle retina and were referred to as dots. In 23 of the cases, these dots corresponded to what has been described as an early hyperfluorescent "wreath-like pattern." The FA also showed multifocal minimally staining lesions in late phases of the angiogram. These lesions were identified in 28 cases in the deep retina and were referred to as spots. In two cases, early hyperfluorescent lesions were present in the foveal area. These lesions were interpreted as "window defect" or transmission of the perfused choriocapillaris through a disturbance in the RPE and macula luteal pigment coinciding with the typical clinically evident macular granularity seen in MEWDS. Optic nerve leakage was also observed as late staining on the FA in 27 patients (Figure 2).

Indocyanine green angiography. Indocyanine green angiography showed the dots to be hypofluorescent in the early- to mid-phases corresponding to the hyperfluorescence seen with FA. The dots seen with ICGA were also localized to the middle retina in 18 cases. In the late stages of the ICGA, larger hypofluorescent lesions were seen in the deep retina. These lesions were identified in all the 22 patients who had ICGA and corresponded to the spots seen with FA. The dots were located anterior to the spots in all of the cases.

Combined hypofluorescent spots with overlying dots ("dots on spots") were observed in the late stages of the ICGA in 18 cases. Dots were not present without spots; however, there were areas that demonstrated spots exclusively with no evidence of dots. In all cases, the number of spots seen with ICGA exceeded those noted clinically or documented with fundus photography. In four cases during the longitudinal period of the study, the spots were observed to disappear before the dots on ICGA and with color photography. The ICGA was the imaging modality that most clearly demonstrated the multifocal spots and a zonal hypofluorescence in the peripapillary area. This hypofluorescence around the disk was observed in 19 of the 22 cases that had ICGA (Figure 3). This corresponded to enlargement of the blind spot area on visual field testing noted in all 13 patients who had this testing.

Spectral domain optical coherence tomography. At the fovea, OCT showed disruption of the ellipsoid zone and accumulations of a hyperreflective material that was of variable size and shape. The reflective material varied from a dome-shaped deposit over the RPE to linear vertical accumulations, and other irregular formations centered at the ellipsoid. All cases were noted to have this hyperreflective material resting on the RPE and extending anteriorly through the interdigitation zone, ellipsoid zone, and ONL toward the inner retina. Irregularities of the RPE were also observed (Figure 4). At the site of extrafoveal spots, OCT imaging also revealed the presence of discontinuities or disruptions centered around the ellipsoid zone to include the interdigitation and sometimes the outer nuclear areas (Figure 5). In 24 patients, the spots seen with FA and ICGA correlated well with this OCT finding (Figure 5, top row). In 13 patients, a vertical extension of the ellipsoid abnormalities could be correlated to the dots seen on FA and ICGA (Figure 5, bottom row). 

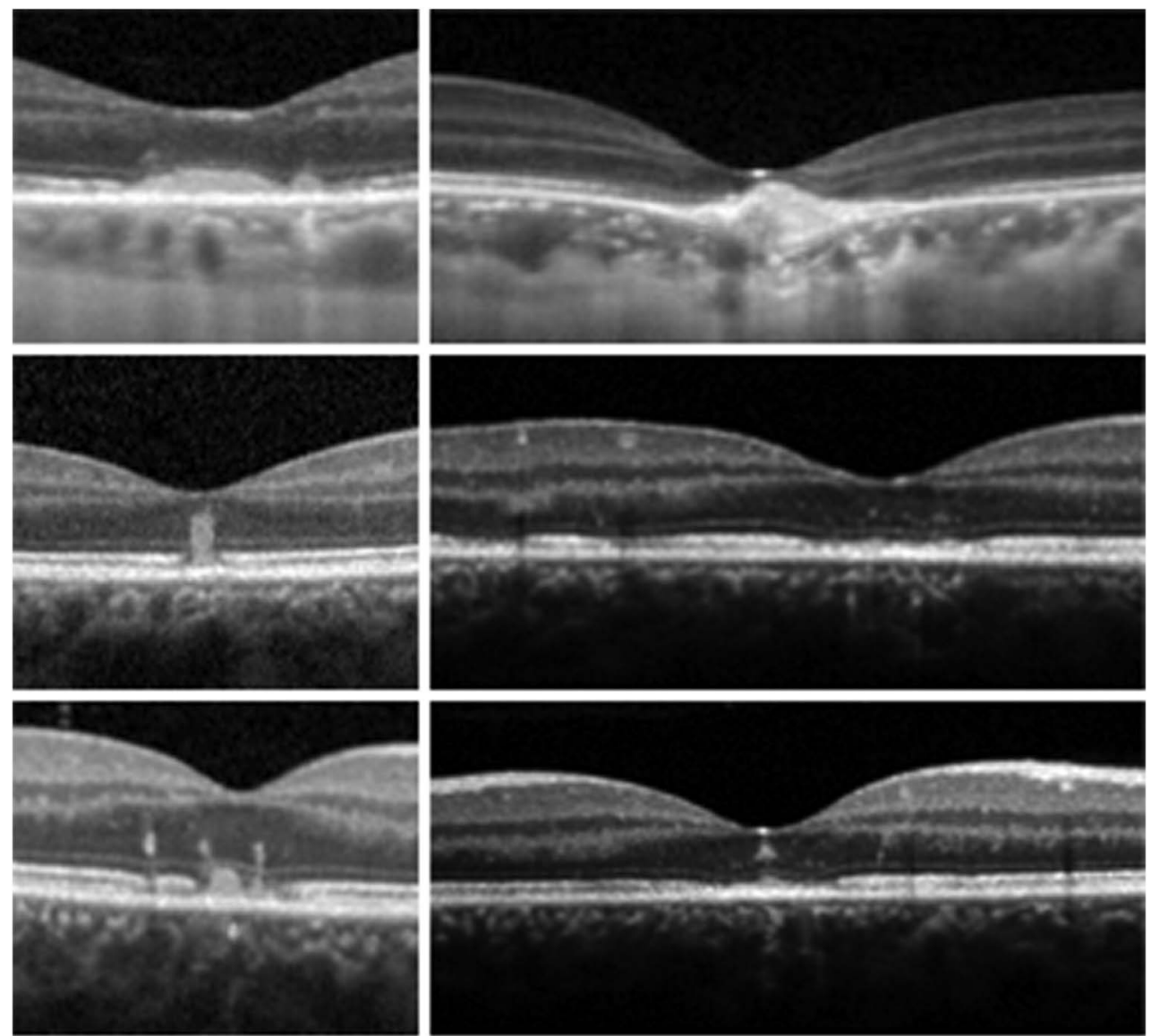

Fig. 4. Optical coherence tomography foveal findings. Hyperreflective material of variable shapes, resting on the RPE and extending anteriorly through the interdigitation zone, ellipsoid zone, and ONL toward the inner retina, was observed. Irregularities of the RPE, disruptions of outer retinal layers, and hyperreflectivity of the ONL were also observed.
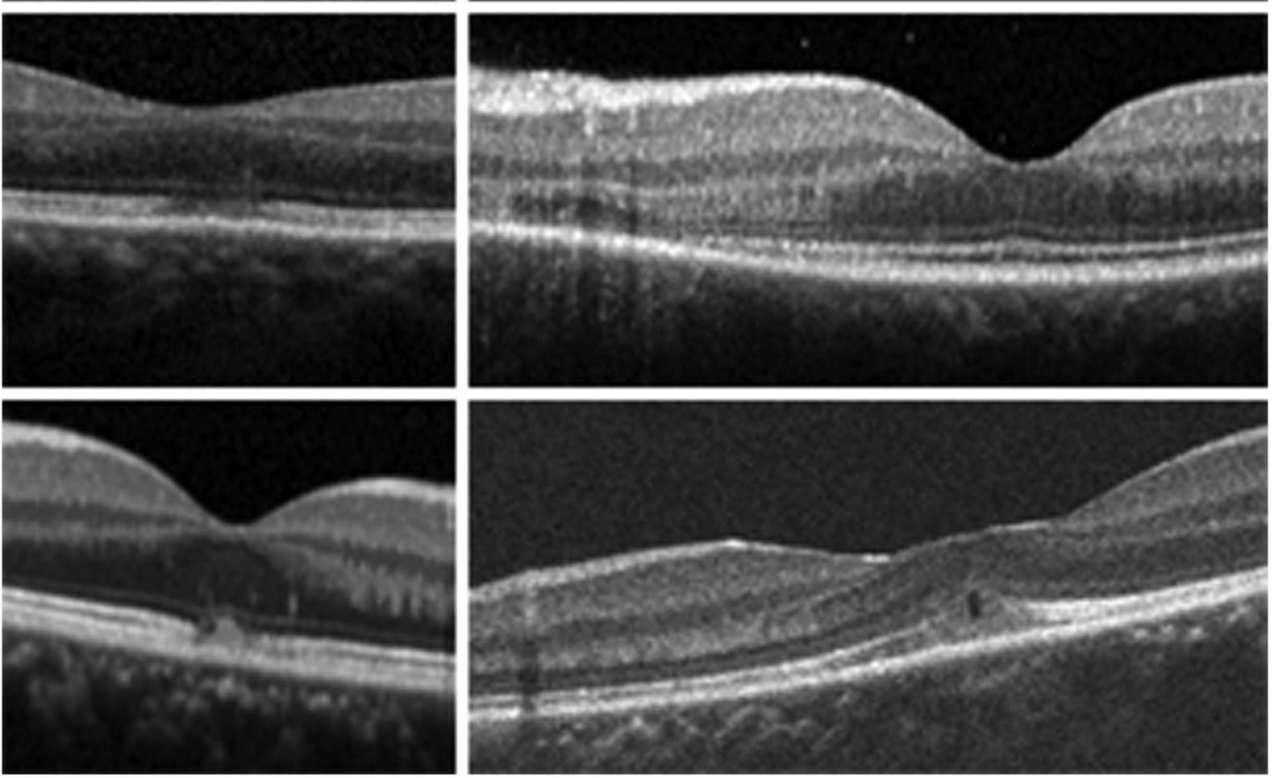

Peripapillary subretinal fluid was present in three patients and was seen as an accumulation of hyporeflective material surrounding the optic nerve with OCT.$^{30}$ One of these cases developed subsequent outer retinal RPE atrophy. Two of the cases that showed peripapillary fluid also had an elevation of the RPE creating a separation between the RPE and Bruch membrane (pigment epithelial detachment) during the acute stage of the diseases. One of these cases concomitantly developed transient intraretinal fluid. In 17 patients, clinically detectable vitreous cells were confirmed with OCT. Persistent peripapillary atrophy/scarring was 

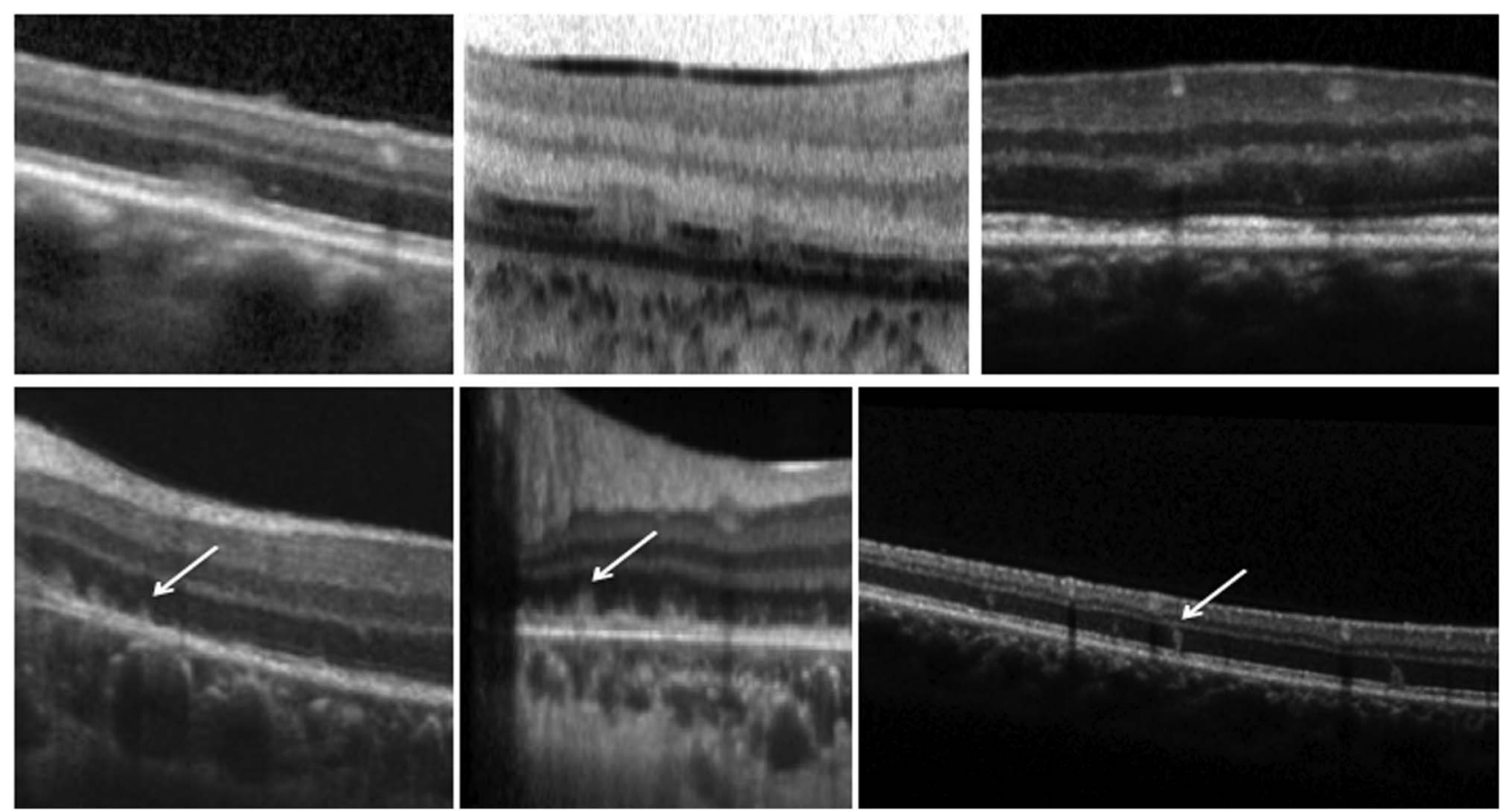

Fig. 5. Extrafoveal OCT findings: spots and dots. Top row: OCT imaging revealed the presence of discontinuities or disruptions centered around the ellipsoid layer to include the interdigitation zone and ONL in the location of the spots seen in angiograms. Bottom row: vertical extension of the ellipsoid abnormalities was correlated to the dots seen on FA and ICGA.

observed with OCT after the resolution of the acute manifestations in eight cases. Two of these cases had evidence of peripapillary atrophy in the fellow eye.

En face OCT imaging analysis of all six cases demonstrated an unremarkable choroid and choriocapillaris. The RPE layer was also unaffected except for a mild mottled appearance in two patients. The ellipsoid and interdigitation zones were disrupted in areas corresponding to spots, as well as to dots in all patients. The en face of the outer portion of the ONL showed reflective material, which corresponded to the dots seen with FA (Figure 6).

Enhanced depth imaging OCT was available in 11 cases, and these images revealed a mean subfoveal choroidal thickness of 282.54 microns at onset and a reduction of the choroidal thickening to 236.0 microns after resolution of the acute manifestations. These measurements suggested a transient choroidal thickening; however, the differences were not statistically significant $(P=0.09)$.

Short-wavelength fundus autofluorescence. Shortwavelength autofluorescence of the foveal area revealed hypo-SW-AF dots that correlated to the OCT foveal accumulations of material in three cases. One of these cases showed hyper-SW-AF lesions in the foveal area.

The analysis of SW-AF of the remainder of these patients in the rest of the fundus showed hyperautofluorescent areas that correlated to spots observed with FA and ICGA, as well as with OCT disruption of the ellipsoid layers.

Near-infrared autofluorescence. Near-infrared autofluorescence was performed in three patients and showed small areas of hypo-NIR-AF. No additional observations useful in characterizing the MEWDS lesion were noted with this imaging modality.

\section{Clinical Course}

All patients experienced recovery as originally described. ${ }^{1}$ Patients improved in a mean interval of 10 weeks from $0.41 \log$ MAR to $0.03 \log$ MAR $(P=$ $0.00)$. The demographic features and presenting symptoms were as previously described (Table 1). However, based on MMI, there were some unusual features in the course in these MEWDS patients. Two patients showed subtle evidence of bilaterality, one with mild ICGA hypofluorescent spots, and another with OCT en face imaging showing a mild ellipsoid disruption. Multifocal chorioretinitis pigmentary scars with atrophy emerged in two patients.

With respect to recurrent disease, three patients experienced one or more recurrences. One patient had 3 recurrences (4 acute episodes) over a period of 50 weeks with full recovery of central acuity each time. Another patient had a recurrent episode of MEWDS 7 years later. At the time of her recurrence, there was evidence of peripheral phlebitis along with several 
MEWDS acute episode
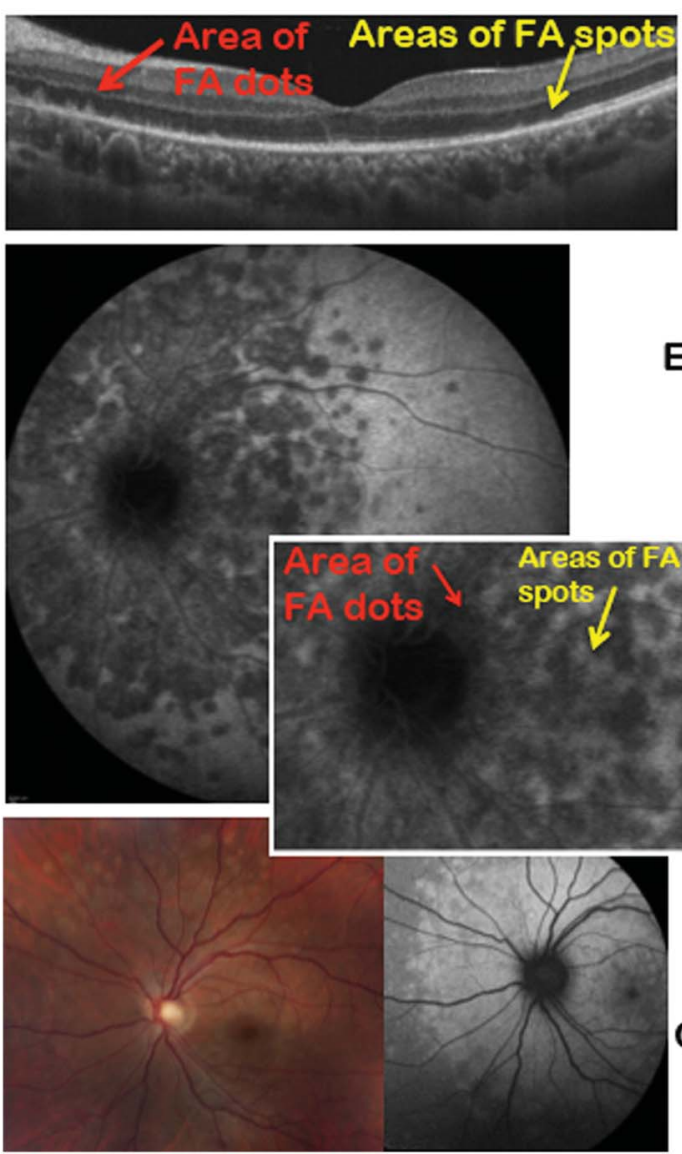

\section{Chor}

Choroid

Fig. 6. En face OCT analysis. Unremarkable choroid and choriocapillaris were observed. The RPE layer was also unaffected in general. The ellipsoid and interdigitation zones were disrupted in areas corresponding to spots and to dots. The en face analysis of the outer portion of the ONL showed a reflective material, which corresponded to the dots seen with angiography. CC, choriocapillaris.

chorioretinitis multifocal atrophic/pigmentary scars. This patient had some clinical features resembling a MFC pattern, but the classical findings of wreathlike on FA and dots on spots as well as confluent zonal ICGA hypofluorescence were indicators most consistent with MEWDS recurrence. The singular case in our series with intraretinal cystic change experienced spontaneous resolution of the cysts without sequel.

\section{Discussion}

Multiple evanescent white dot syndrome has been known as a distinct clinical entity for more than 30 years since the initial description by Jampol et $\mathrm{al}^{1}$ in 1984. The pathogenesis is still unknown, although it has been suggested that it is an immune-mediated process, possibly in genetically predisposed individuals. ${ }^{31}$ At present, there is no conclusive evidence that immunity or genetics play a role. Although gender (female), age (young), and refraction (myopia) seem to be predisposing factors, there are no known racial predilections as the disease has been described in white, Asian, Hispanic, and African American patients. In its typical form, a young adult myopic female presents with a unilateral disturbance of the central vision often with an enlargement of the blind spot. The condition is associated with three consistent clinical findings: 1) multiple, grayish white evanescent lesions scattered in the fundus, 2) granularity in the fovea, and 3) a variably edematous appearing optic nerve. Foveal granularity is present in most cases and, when seen, determines the diagnosis. The number of white spots is very variable, and in fact they may be absent. ${ }^{32}$

Some investigators have suggested that MEWDS is a choroidal vascular disease. Others have considered it to be a disease of the RPE or outer retina. We have no evidence of choroidopathy, but we have seen, in some patients, nonspecific choroidal thickening, which is common in many chorioretinal inflammatory diseases. ${ }^{33}$ There was no evidence of primary RPE disease in this study. However, some nonspecific 
Table 1. Demographic characteristics of the study population and initial symptoms

\begin{tabular}{|c|c|c|c|c|c|c|c|c|c|}
\hline \multirow[b]{2}{*}{$\begin{array}{l}\text { Patient } \\
\text { ID }\end{array}$} & \multirow[b]{2}{*}{$\begin{array}{l}\text { Age at } \\
\text { Onset, } \\
\text { years }\end{array}$} & \multirow[b]{2}{*}{ Gender } & \multirow[b]{2}{*}{$\begin{array}{l}\text { Affected } \\
\text { Eye }\end{array}$} & \multirow[b]{2}{*}{$\begin{array}{l}\text { Initial VA, } \\
\text { logMAR }\end{array}$} & \multirow[b]{2}{*}{$\begin{array}{l}\text { Final VA, } \\
\text { logMAR }\end{array}$} & \multirow[b]{2}{*}{ Refraction } & \multicolumn{3}{|c|}{ Initial Symptoms } \\
\hline & & & & & & & Photopsia & $\begin{array}{c}\text { Complaint of } \\
\text { Central Visual } \\
\text { Loss }\end{array}$ & $\begin{array}{l}\text { Field } \\
\text { Defect at } \\
\text { Onset }\end{array}$ \\
\hline 1 & 14 & $\mathrm{~F}$ & OS & 2.30 & 0.60 & Myopic & Yes & Yes & Yes \\
\hline 2 & 25 & $\mathrm{~F}$ & OS & 1.00 & $\mathrm{n} / \mathrm{a}$ & $\mathrm{n} / \mathrm{a}$ & No & Yes & No \\
\hline 3 & 40 & $\mathrm{~F}$ & OD & 0.40 & 0.00 & Myopic & No & Yes & No \\
\hline 4 & 30 & $\mathrm{~F}$ & OS & 0.10 & 0.00 & Myopic & Yes & Yes & No \\
\hline 5 & 30 & $\mathrm{~F}$ & OD & 0.60 & 0.00 & $\mathrm{n} / \mathrm{a}$ & No & Yes & No \\
\hline 6 & 36 & $\mathrm{~F}$ & OD & 0.00 & 0.00 & Myopic & Yes & Yes & Yes \\
\hline 7 & 49 & $\mathrm{~F}$ & OS & 0.30 & 0.10 & Myopic & No & No & No \\
\hline 8 & 20 & M & OD & 0.70 & $\mathrm{n} / \mathrm{a}$ & Emmetropic & $\mathrm{n} / \mathrm{a}$ & $\mathrm{n} / \mathrm{a}$ & $\mathrm{n} / \mathrm{a}$ \\
\hline 9 & 26 & $\mathrm{~F}$ & OD & 0.70 & 0.00 & $\mathrm{n} / \mathrm{a}$ & $\mathrm{n} / \mathrm{a}$ & Yes & $\mathrm{n} / \mathrm{a}$ \\
\hline 10 & 25 & $\mathrm{~F}$ & OD & 0.30 & $\mathrm{n} / \mathrm{a}$ & $\mathrm{n} / \mathrm{a}$ & Yes & Yes & No \\
\hline 11 & 26 & $\mathrm{~F}$ & OS & 0.00 & $\mathrm{n} / \mathrm{a}$ & Myopic & $\mathrm{n} / \mathrm{a}$ & $\mathrm{n} / \mathrm{a}$ & Yes \\
\hline 12 & 24 & $\mathrm{~F}$ & OS & 0.40 & 0.00 & $\mathrm{n} / \mathrm{a}$ & Yes & Yes & $\mathrm{n} / \mathrm{a}$ \\
\hline 13 & 27 & $\mathrm{~F}$ & OS & 0.54 & $\mathrm{n} / \mathrm{a}$ & $\mathrm{n} / \mathrm{a}$ & Yes & Yes & Yes \\
\hline 14 & 25 & M & OD & 0.10 & $\mathrm{n} / \mathrm{a}$ & Myopic & Yes & Yes & Yes \\
\hline 15 & 32 & $M$ & OD & 0.54 & 0.00 & Myopic & $\mathrm{n} / \mathrm{a}$ & Yes & $\mathrm{n} / \mathrm{a}$ \\
\hline 16 & 46 & M & OS & 0.60 & 0.10 & $\mathrm{n} / \mathrm{a}$ & $\mathrm{n} / \mathrm{a}$ & Yes & $\mathrm{n} / \mathrm{a}$ \\
\hline 17 & 34 & $\mathrm{~F}$ & OS & 0.18 & $\mathrm{n} / \mathrm{a}$ & $\mathrm{n} / \mathrm{a}$ & Yes & Yes & No \\
\hline 18 & 37 & $\mathrm{~F}$ & OS & 0.70 & $\mathrm{n} / \mathrm{a}$ & $\mathrm{n} / \mathrm{a}$ & $\mathrm{n} / \mathrm{a}$ & $\mathrm{n} / \mathrm{a}$ & $\mathrm{n} / \mathrm{a}$ \\
\hline 19 & 36 & $\mathrm{~F}$ & OS & 0.00 & $\mathrm{n} / \mathrm{a}$ & $\mathrm{n} / \mathrm{a}$ & Yes & No & Yes \\
\hline 20 & 23 & $\mathrm{~F}$ & OS & 0.00 & 0.00 & Myopic & Yes & Yes & Yes \\
\hline 21 & 33 & M & OS & 0.60 & $\mathrm{n} / \mathrm{a}$ & Myopic & Yes & Yes & No \\
\hline 22 & 26 & M & Os & 0.40 & $\mathrm{n} / \mathrm{a}$ & Myopic & No & Yes & No \\
\hline 23 & 16 & $\mathrm{~F}$ & OD & 0.00 & 0.00 & Myopic & Yes & Yes & $\mathrm{n} / \mathrm{a}$ \\
\hline 24 & 19 & M & os & 0.48 & 0.00 & Emmetropic & No & Yes & Yes \\
\hline 25 & 48 & $\mathrm{~F}$ & OS & 0.00 & $\mathrm{n} / \mathrm{a}$ & Myopic & No & Yes & Yes \\
\hline 26 & 27 & $\mathrm{~F}$ & OS & 0.00 & 0.00 & Myopic & Yes & No & Yes \\
\hline 27 & 24 & $\mathrm{~F}$ & OD & 0.4 & 0.00 & Myopic & No & Yes & Yes \\
\hline 28 & 36 & $\mathrm{~F}$ & OD & 0.5 & 0.00 & $\mathrm{n} / \mathrm{a}$ & No & Yes & No \\
\hline 29 & 27 & $\mathrm{~F}$ & OD & 0.2 & 0.00 & $\mathrm{n} / \mathrm{a}$ & Yes & Yes & No \\
\hline 30 & 18 & $\mathrm{~F}$ & OD & 0.6 & -0.10 & Myopic & Yes & Yes & Yes \\
\hline 31 & 16 & $M$ & OD & 0.5 & -0.10 & Myopic & No & Yes & No \\
\hline 32 & 30 & $\mathrm{~F}$ & OS & 0.6 & 0.00 & Myopic & No & Yes & Yes \\
\hline 33 & 27 & $\mathrm{~F}$ & OS & 0.30 & 0.10 & Emmetropic & No & Yes & $\mathrm{n} / \mathrm{a}$ \\
\hline 34 & 26 & $\mathrm{~F}$ & OS & 0.17 & $\mathrm{n} / \mathrm{a}$ & $\mathrm{n} / \mathrm{a}$ & $\mathrm{n} / \mathrm{a}$ & $\mathrm{n} / \mathrm{a}$ & $n / a$ \\
\hline
\end{tabular}

n/a, not available; OD, right eye; OS, left eye; VA, visual acuity.

secondary mottling and proliferation of the RPE were detected.

The clinical manifestations in MEWDS in our series were in general consistent with the ophthalmic literature (Table 1). The disorder is unilateral in almost all of the cases. We found evidence of bilaterality in only two patients based on ICGA and en face OCT imaging. Previous reports have described bilaterality, ${ }^{1,4,7-22}$ although the involvement of the second eye was mild. Li and Kishi ${ }^{19}$ performed functional tests such as multifocal electroretinogram and full-field electroretinogram, proving bilateral dysfunction of photoreceptors in most of the MEWDS cases they studied. Second, frequent optic nerve involvement was also consistent with the literature. In our series, no patient developed choroidal neovascularization, although this has been reported. ${ }^{23,24}$
Our report serves also to reconcile some of the issues regarding differences between MFC and MEWDS. It is well known that MEWDS and MFC have similar demographics (usually young myopic females) and can resemble each other (enlarged blind spot, transient white lesions, late scars). With MMI analysis, differentiation of the two entities is facilitated by the presence of early hyperfluorescent wreathlike dots on FA, hypofluorescent plaques on ICGA and foveal granularity on the clinical examination, color photography, and OCT in MEWDS. One example of OCT distinguishing finding is the ellipsoid degeneration that extends through the inner aspect of the RPE and the interdigitation line to exhibit a hyperreflective material that protrudes toward the ONL and beyond in MEWDS. In contrast, OCT imaging in MFC shows disruptions of the Bruch membrane/RPE complex and 
hyperreflective material accumulating under the RPE and expanding into the retina. ${ }^{34}$ The cases seen herein had little resemblance to acute zonal occult outer retinopathy ${ }^{35}$ or acute macular neuroretinopathy.

Multimodal imaging in our MEWDS series also provides a better understanding of the pathogenesis of the disorder. The classic wreathlike retinal lesion best seen on FA may be due to middle or deep retinal capillary hyperfluorescence, perhaps from dilation of the retinal microcirculation by inflammation. Inflammatory mediators may extend from the outer to the middle retina to affect the deep retinal capillaries. Another explanation for the early hyperfluorescence of these lesions on FA may relate to the excitation of microglia. Microglia have been implicated in immune-mediated inflammatory processes of the retina. ${ }^{36}$ The stellate configuration of those cells in the retina resembles the wreathlike lesions seen on FA in MEWDS. Similar observations have been made in transgenic mice with a fluorescent protein. ${ }^{36}$ Activation of the microglia by the inflammation in conjunction with dilation of the deeper retinal circulation may contribute to the presenting signs of MEWDS.

Our series reports on the use of MMI for MEWDS patients, establishing more distinguishing features that help in differentiating the disorder from other idiopathic chorioretinal inflammatory diseases. The use of MMI has also recently characterized these diseases, including acute zonal occult outer retinopathy, ${ }^{35}$ idiopathic MFC (also known as punctate inner choroiditis), ${ }^{37}$ persistent posterior maculopathy, ${ }^{38}$ and acute macular neuroretinopathy. ${ }^{39}$

Finally, the use of new imaging technologies has provided new standards for diagnosing MEWDS with an array of clinical manifestations and imaging findings seen in our series. Our findings implicate a disease of the photoreceptors, which is almost completely reversible. The RPE and choroid were only secondarily and transiently involved.

\section{Limitations}

Although this is the largest series to date, the number of MEWDS patients was small. The study was retrospective. Most importantly, there is an absence of histopathologic correlation. As expected in such a study, not all the patients had imaging with all modalities. In addition, localization and nature of the dots will require future research, possibly using future emerging systems and phase-based or intensitybased OCT angiography for three-dimensional mapping of the middle retina, where the dots reside as identified with stereo-photography and FA.

\section{Summary}

The diagnosis of MEWDS is made in healthy young patients usually female, based on the presence of multiple gray-white evanescent lesions scattered in the fundus, granularity in the fovea, swelling of the optic nerve, and clinical course with invariable functional recovery. Angiography demonstrates characteristic findings: FA frequently shows mid retinal early fluorescent dots distributed in a wreathlike pattern, which correlate to fundus photography, fundus autofluorescence, and ICGA. Imaging with ICGA shows the dots and also hypofluorescent, deeper, and larger spots, which are occasionally confluent, demonstrating a defining large plaque of deep retinal hypofluorescence. Optical coherence tomography imaging shows multifocal debris centered at and around the ellipsoid layer corresponding to the location of spots seen with photography, ICGA, and FA. Protrusions of the hyperreflectant material from the ellipsoid layer toward the ONL correspond to the location of dots that are seen with photography, ICGA, and FA. A plaque of outer retinal involvement from confluent spots appears to be pathognomonic of MEWDS, differentiating it from idiopathic MFC. We conclude that MEWDS is predominantly a disease of the outer retina, centered at the ellipsoid zone, but also involving the interdigitation zone and the ONL. Minor changes in the acute and healed stages also occur at the RPE and choroid.

Key words: multiple evanescent white dot syndrome, MEWDS, multimodal imaging, retina, uveitis, posterior uveitis, autofluorescence, optical coherence tomography, fluorescein angiography, indocyanine green angiography.

\section{References}

1. Jampol LM, Sieving PA, Pugh D, et al. Multiple evanescent white dot syndrome. I. Clinical findings. Arch Ophthalmol 1984;102:671-674.

2. Obana A, Kusumi M, Miki T. Indocyanine green angiographic aspects of multiple evanescent white dot syndrome. Retina 1996;16:97-104.

3. Gross NE, Yannuzzi LA, Freund KB, et al. Multiple evanescent white dot syndrome. Arch Ophthalmol 2006;124:493-500.

4. Nguyen MH, Witkin AJ, Reichel E, et al. Microstructural abnormalities in MEWDS demonstrated by ultrahigh resolution optical coherence tomography. Retina 2007;27:414-418.

5. Yenerel NM, Kucumen B, Gorgun E, Dinc UA. Atypical presentation of multiple evanescent white dot syndrome (MEWDS). Ocul Immunol Inflamm 2008;16:113-115.

6. Furino C, Boscia F, Cardascia N, et al. Fundus autofluorescence and multiple evanescent white dot syndrome. Retina 2009;29:60-63.

7. Aaberg TM, Campo RV, Joffe L. Recurrences and bilaterality in the multiple evanescent white-dot syndrome. Am J Ophthalmol 1985;100:29-37. 
8. Meyer RJ, Jampol LM. Recurrences and bilaterality in the multiple evanescent white-dot syndrome. Am J Ophthalmol 1986;101:388-389.

9. Jost BF, Olk RJ, McGaughey A. Bilateral symptomatic multiple evanescent white-dot syndrome. Am J Ophthalmol 1986; 101:489-490.

10. Nakao K, Isashiki M. Multiple evanescent white dot syndrome. Jpn J Ophthalmol 1986;30:376-384.

11. Chung YM, Yeh TS, Liu JH. Increased serum IgM and IgG in the multiple evanescent white-dot syndrome. Am J Ophthalmol 1987;104:187-188.

12. Gass JD, Hamed LM. Acute macular neuroretinopathy and multiple evanescent white dot syndrome occurring in the same patients. Arch Ophthalmol 1989;107:189-193.

13. Kimmel AS, Folk JC, Thompson HS, Strnad LS. The multiple evanescent white-dot syndrome with acute blind spot enlargement. Am J Ophthalmol 1989;107:425-426.

14. Borruat FX, Othenin-Girard P, Safran AB. Multiple evanescent white dot syndrome. Klin Monbl Augenheilkd 1991; 198:453-456.

15. Tsai L, Jampol LM, Pollock SC, Olk J. Chronic recurrent multiple evanescent white dot syndrome. Retina 1994;14: 160-163.

16. Reddy CV, Brown J Jr., Folk JC, et al. Enlarged blind spots in chorioretinal inflammatory disorders. Ophthalmology 1996; 103:606-617.

17. Asano T, Kondo M, Kondo N, et al. High prevalence of myopia in Japanese patients with multiple evanescent white dot syndrome. Jpn J Ophthalmol 2004;48:486-489.

18. Fernández-Barrientos Y, Díaz-Valle D, Méndez-Fernández R, Benítez-Del-Castillo JM. Possible recurrent multiple evanescent white dot syndrome and chroroidal neovascularization. Arch Soc Esp Oftalmol 2007;82:587-590.

19. Li D, Kishi S. Restored photoreceptor outer segment damage in multiple evanescent white dot syndrome. Ophthalmology 2009; 116:762-770.

20. Dell'Omo R, Mantovani A, Wong R, et al. Natural evolution of fundus autofluorescence findings in multiple evanescent white dot syndrome: a long-term follow-up. Retina 2010; 30:1479-1487.

21. Abu-Yaghi NE, Hartono SP, Hodge DO, et al. White dot syndromes: a 20-year study of incidence, clinical features, and outcomes. Ocul Immunol Inflamm 2011;19:426-430.

22. Takeda M, Kimura S, Tamiya M. Acute disseminated retinal pigment epitheliopathy. Folia Ophthalmol Jpn 1984;35: 2613-2620.

23. Callanan D, Gass JD. Multifocal choroiditis and choroidal neovascularization associated with the multiple evanescent white dot and acute idiopathic blind spot enlargement syndrome. Ophthalmology 1992;99:1678-1685.
24. Wyhinny GJ, Jackson JL, Jampol LM, Caro NC. Subretinal neovascularization following multiple evanescent white-dot syndrome. Arch Ophthalmol 1990;108:1384-1385.

25. Hernández Artola F, Garay Aramburu G, Llorca Pellicer S. Multiple evanescent white-dot syndrome: bilateralization four years later [in Spanish]. Arch Soc Esp Oftalmol 2002; 77:393-396.

26. Boretsky A, Mirza S, Khan F, et al. High-resolution multimodal imaging of multiple evanescent white dot syndrome. Ophthalmic Surg Lasers Imaging Retina 2013;44:296-300.

27. Bryan RG, Freund KB, Yannuzzi LA, et al. Multiple evanescent white dot syndrome in patients with multifocal choroiditis. Retina 2002;22:317-322.

28. Spaide RF, Koizumi H, Pozzoni MC. Enhanced depth imaging spectral-domain optical coherence tomography. Am J Ophthalmol 2008;146:496-500.

29. Margolis R, Spaide RF. A pilot study of enhanced depth imaging optical coherence tomography of the choroid in normal eyes. Am J Ophthalmol 2009;147:811-815.

30. Chao DL, Marsiglia M, Ahmad B, et al. Peripapillary serous detachment in multiple evanescent white dot syndrome. Retina 2015;35:521-524.

31. Jampol LM, Becker KG. White spot syndromes of the retina: a hypothesis based on the common genetic hypothesis of autoimmune/inflammatory disease. Am J Ophthalmol 2003;135: 376-379.

32. Shelsta H, Rao RR, Bhatt HK, Jampol LM. Atypical presentations of multiple evanescent white dot syndrome (MEWDS) without white dots: a case series. Retina 2011;34:973-976.

33. Aoyagi R, Hayashi T, Masai A, et al. Subfoveal choroidal thickness in multiple evanescent white dot syndrome. Clin Exp Optom 2012;95:212-217.

34. Spaide RF, Goldberg N, Freund KB. Redefining multifocal choroiditis and panuveitis and punctate inner choroidopathy through multimodal imaging. Retina 2013;33:1315-1324.

35. Mrejen S, Khan S, Gallego-Pinazo R, et al. Acute zonal occult outer retinopathy: a classification based on multimodal imaging. JAMA Ophthalmol 2014;132:1089-1098.

36. Wang M, Wang X, Zhao L, et al. Macroglia-microglia interactions via TSPO signaling regulates microglial activation in the mouse retina. J Neurosci 2014;34:3793-3806.

37. Jung JJ, Khan S, Mrejen S, et al. Idiopathic multifocal choroiditis with outer retinal or chorioretinal atrophy. Retina 2014; 34:1439-1450.

38. Gendy MG, Fawzi AA, Wendel RT, et al. Multimodal imaging in persistent placoid maculopathy. JAMA Ophthalmol 2014; 132:38-44.

39. Yzer S, Freund KB, Engelbert M. Imaging in the diagnosis and management of acute macular neuroretinopathy. Int Ophthalmol Clin 2012;52:269-273. 